\title{
Rentabilidad financiera y liquidez corriente de las empresas del sector industrial que negocian en la Bolsa de Valores de Lima, 2011-2014
}

\section{Financial profitability and current liquidity of companies in the industrial sector that trade on the Lima Stock Exchange, 2011-2014}

\author{
Contreras Unchupaico, Rónald Roy; Palacios Ponce de León, Francis David \\ EP de Contabilidad, Facultad de Ciencias Empresariales, Universidad Peruana Unión \\ Recibido 14 de setiembre del 2015-Aceptado 6 de diciembre del 2015
}

\begin{abstract}
Resumen
El objetivo de esta investigación es determinar la relación entre rentabilidad financiera y liquidez corriente de las empresas del sector industrial que negocian en la Bolsa de Valores de Lima, periodos, 2011-2014. Es un estudio de diseño no experimental de tipo retrospectivo. La población está compuesta por 29 empresas del sector industrial que negocian en la Bolsa de Valores de Lima. El análisis descriptivo tuvo los siguientes resultados: a) La distribución de las empresas (41\%) se encuentra en un nivel medio de rentabilidad financiera de los datos procesados de los períodos 2011-2014. b) La distribución de las empresas por períodos se da de la siguiente manera: (41\%) se encuentra en un nivel alto de rentabilidad financiera en el período $2011,(48 \%)$ se encuentra en un nivel medio de rentabilidad financiera en el período 2012, (41\%) se encuentra en un nivel bajo de rentabilidad financiera en el período 2013 y (41\%) se encuentra en un nivel medio de rentabilidad financiera en el período 2014, c) La distribución de las empresas por períodos se da de la siguiente manera: (39\%) se encuentra en un nivel medio de liquidez corriente de los datos procesados de los períodos (2011-2014). La distribución de las empresas (48\%) se encuentra en un nivel medio de liquidez corriente en el período 2011, (34\%) se encuentra en un nivel alto de liquidez corriente en el período 2012, (45\%) se encuentra en un nivel medio de liquidez corriente en el período 2013, (45\%) se encuentra en un nivel bajo de liquidez corriente en el período 2014. Existe relación $(\mathrm{r}=-0.218 ; \mathrm{p}=0.019)$ entre la rentabilidad financiera y la liquidez corriente.
\end{abstract}

Palabras clave: Rentabilidad financiera, liquidez corriente, bolsa de valores, industria.

\begin{abstract}
The aim of this research is to determine the relationship between financial profitability and current liquidity of the companies of the industrial sector that trade in the Lima Stock Exchange, periods, 2011-2014. It is a retrospective non-experimental design study. The population is composed of 29 companies of the industrial sector that negotiate in the Stock Exchange of Lima. The descriptive analysis had the following results: a) The distribution of the companies (41\%), are in an average level of financial profitability of the processed data of the periods 2011-2014. B) The distribution of companies by periods is as follows: (41\%) are in a high level of financial profitability in $2011,(48 \%)$ are in an average level of financial profitability in the period $2012,(41 \%)$ are in a low level of financial profitability in the period 2013 and (41\%) are in an average level of financial profitability in the period 2014, c) The distribution of companies by periods occurs The following way: (39\%) are at an average level of current liquidity of the processed data of the periods (2011-2014). The distribution of companies (48\%) are in an average level of current liquidity in 2011, (34\%) are in a high level of current liquidity in the period 2012, (45\%) are in a level average liquidity in the period 2013, (45\%) are at a low level of current liquidity in the period 2014. There is a relation $(\mathrm{r}=-0.218 ; \mathrm{p}=0.019)$ between financial profitability and current liquidity.
\end{abstract}

Keywords: Financial profitability, current liquidity, stock exchange, industry.

Correspondencia al autor:

email: ronaldroyc@gmail.com, davidpalacios100914@gmail.com 


\section{Introducción}

Gajardo Bahamonde y Saldivia Díaz (2013) sostienen que la información sobre indicadores financieros, por sectores, son de gran utilidad en términos de rendimiento y competitividad, de igual forma para la gestión y toma de decisiones en la empresa.

La gerencia, en algún momento, toma decisiones importantes acorde a cada situación de la empresa. En ese sentido, dentro del marco de liquidez corriente y rentabilidad financiera, la gerencia empresarial cuenta con escasa información de los criterios, estándares y patrones que guían para decidir sobre qué aspecto es conveniente priorizar. Por ello la pregunta ¿Será ideal enfocarse netamente en generar liquidez corriente? o ¿El escenario principal debe ser dirigido a obtener rentabilidad financiera? Considerando que una de las preocupaciones importantes de la empresa es cubrir sus obligaciones a corto plazo y de los accionistas se enfoca en la obtención de la mayor rentabilidad posible.

García (2014) asegura la existencia de una relación inversa entre la rentabilidad y liquidez. En la referencia, alcanzar rentabilidad no representa adquirir liquidez o la disponibilidad del efectivo en el momento, como resultado se convierte en una preocupación considerable para la gerencia, cuando la junta general de accionistas decide la distribución de los dividendos obtenidos en un cierto periodo determinado.

El presente trabajo proporcionará información a la gerencia sobre los comportamientos y las características sobre los niveles de la rentabilidad financiera y liquidez corriente de las empresas que negocian en la bolsa de valores de Lima del sector industrial, asimismo, permitirá respaldar futuras investigaciones relacionadas al tema de investigación.

Se realizó el estudio con el objetivo de determinar la relación entre la rentabilidad financiera y la liquidez corriente, así también determinar la cantidad de empresas según los niveles de rentabilidad financiera y determinar la cantidad de empresas según los niveles de liquidez corriente, en las empresas del sector industrial que negocian en la Bolsa de Valores de Lima, 2011-2014.

\section{Método}

La presente es una investigación no experimental de diseño retrospectivo, puesto que este estudio se reali- za con la información de años anteriores publicados en la Bolsa de Valores de Lima, sin la manipulación ni cambios de los datos recolectados. El enfoque de esta investigación se basa en el análisis descriptivo correlacional porque, mediante el coeficiente de correlación, se podrá concluir la relación que tiene la rentabilidad financiera y la liquidez corriente, y es cuantitativo porque mide las variables de estudio y aplica el análisis estadístico.

Los datos de las empresas que fueron analizados pertenecen al sector industrial, estos fueron publicados en la página web de la Bolsa de Valores de Lima 2011-2014. La información de los estados financieros publicados en la página oficial de la Bolsa de Valores de Lima.

Los datos fueron analizados mediante estadísticos descriptivos como porcentajes y estadísticos inferenciales como el $r$ de Pearson para determinar el comportamiento y la correlación de las variables rentabilidad financiera y liquidez corriente.

\section{Resultados y Discusión}

Los resultados del análisis descriptivo de las empresas industriales según nivel de rentabilidad financiera, cantidad de empresas industriales según nivel de liquidez corriente y correlación de rentabilidad financiera y liquidez corriente, se presentan en las siguientes tablas:

Tabla 1

Cantidad de empresas industriales según nivel de rentabilidad financiera de las empresas que negocian en la Bolsa de Valores de Lima en los períodos 2011-2014

\begin{tabular}{ccc}
\hline Nivel & $\mathbf{N}$ & $\mathbf{\%}$ \\
\hline Bajo & 35 & 30 \\
Medio & 47 & 41 \\
Alto & 34 & 29 \\
\hline Total & 116 & 100 \\
\hline
\end{tabular}

Según se aprecia, en la tabla 1, la distribución de empresas se encuentra entre el 30\% y el $41 \%$, resaltándose el nivel medio con un $41 \%$ de los datos procesados pertenecientes a los períodos 2011 - 2014. 
Rentabilidad financiera y liquidez corriente de las empresas del sector industrial que negocian en la Bolsa de Valores de Lima, 2011-2014

Tabla 2

Cantidad de empresas industriales según nivel de rentabilidad financiera comparativo de las empresas que negocian en la Bolsa de Valores de Lima en los períodos 2011-2014

\begin{tabular}{ccccc}
\hline \multirow{2}{*}{ Nivel } & \multicolumn{5}{c}{$\%$} \\
\cline { 2 - 5 } & $\mathbf{2 0 1 1}$ & $\mathbf{2 0 1 2}$ & $\mathbf{2 0 1 3}$ & $\mathbf{2 0 1 4}$ \\
\hline Bajo & $\mathbf{1 7}$ & $\mathbf{2 1}$ & $\mathbf{4 1}$ & $\mathbf{4 1}$ \\
Medio & 41 & 48 & 31 & 41 \\
Alto & 41 & 31 & 28 & 17 \\
\hline Total & 100 & 100 & 100 & 100 \\
\hline
\end{tabular}

Referente a la tabla 2, se observa que en el nivel bajo existe un comportamiento de fluctuación en la cantidad de empresas, es decir, cada año más empresas disminuyeron su rentabilidad financiera $\mathrm{y}$, por ende, se situaron en este nivel.

Tabla 3

Cantidad de empresas industriales según nivel de liquidez corriente de las empresas que negocian en la Bolsa de Valores de Lima en los períodos 2011-2014

\begin{tabular}{ccc}
\hline Nivel & $\mathbf{N}$ & $\mathbf{\%}$ \\
\hline Bajo & 36 & 31 \\
Medio & 45 & 39 \\
Alto & 35 & 30 \\
\hline Total & 116 & 100 \\
\hline
\end{tabular}

Según se muestra en la tabla 3, la distribución de empresas se encuentra en un nivel bajo con un 31\% y un nivel alto con un $30 \%$, resaltándose el nivel medio con un 39\% de los datos procesados en los períodos $2011-2014$.

Tabla 4

Cantidad de empresas industriales según nivel de liquidez corriente comparativo de las empresas que negocian en la Bolsa de Valores de Lima en los períodos 2011-2014

\begin{tabular}{ccccc}
\hline \multirow{2}{*}{ Nivel } & \multicolumn{5}{c}{$\%$} \\
\cline { 2 - 5 } & $\mathbf{2 0 1 1}$ & $\mathbf{2 0 1 2}$ & $\mathbf{2 0 1 3}$ & $\mathbf{2 0 1 4}$ \\
\hline Bajo & 21 & 31 & 28 & 45 \\
Medio & 48 & 34 & 45 & 28 \\
Alto & 31 & 34 & 28 & 28 \\
\hline Total & 100 & 100 & 100 & 100 \\
\hline
\end{tabular}

Como se aprecia, en la tabla 4, existe un aumento en la cantidad de empresas en el nivel bajo cada 2 períodos, es decir, del 2011 al 2012 se observa que este nivel se incrementa y del 2012 al 2013 disminuye la cantidad de empresas y para el 2014 nuevamente se refleja el aumento en este nivel.
Tabla 5

Análisis de correlación de rentabilidad financiera y liquidez corriente en los períodos 2011-2014

\begin{tabular}{lccc}
\hline & \multicolumn{3}{c}{ Liquidez Corriente } \\
\cline { 2 - 4 } & $\mathrm{r}$ & $\mathrm{P}$ & $\mathrm{N}$ \\
\hline $\begin{array}{l}\text { Rentabilidad } \\
\text { Financiera }\end{array}$ & -.218 & .019 & 29 \\
\hline
\end{tabular}

Se observa, en la tabla 5 , el coeficiente de correlación según Pearson es $r=-.218$ que indica la existencia de una correlación negativa inversa y $\mathrm{p}=.019$, lo que señala una correlación significativa. Ello quiere decir que a mayores niveles de rentabilidad financiera, los niveles de liquidez corriente serán menores en las empresas analizadas que publicaron información financiera en la BVL en los períodos 2011-2014, esto también se aplica viceversa.

Tabla 6

Análisis de correlación entre rentabilidad financiera y liquidez corriente en el período 2011

\begin{tabular}{lccc}
\hline & \multicolumn{4}{c}{ Liquidez Corriente } \\
\hline & $\mathrm{r}$ & $\mathrm{P}$ & $\mathrm{N}$ \\
\hline $\begin{array}{l}\text { Rentabilidad } \\
\text { Financiera }\end{array}$ & -.432 & .019 & 29 \\
\hline
\end{tabular}

Se observa, en la tabla 6 , el coeficiente de correlación según Pearson es $r=-.432$ que indica la existencia de una correlación negativa inversa y $\mathrm{p}=.019$ lo que señala una correlación significativa. Ello quiere decir que a mayores niveles de rentabilidad financiera, los niveles de liquidez corriente serán menores en las empresas analizadas que publican información financiera en la BVL en el período 2011, esto también se aplica viceversa.

Tabla 7

Análisis de correlación entre rentabilidad financiera y liquidez corriente en el período 2012

\begin{tabular}{lccc}
\hline & \multicolumn{3}{c}{ Liquidez Corriente } \\
\cline { 2 - 4 } & $\mathrm{r}$ & $\mathrm{P}$ & $\mathrm{N}$ \\
\hline $\begin{array}{l}\text { Rentabilidad } \\
\text { Financiera }\end{array}$ & -.289 & .128 & 29 \\
\hline
\end{tabular}

Se observa, en la tabla 7, el coeficiente de correlación según Pearson es $r=-.289$ que indica la existencia de una correlación negativa inversa y $\mathrm{p}=.128$ lo que señala una correlación no significativa. Ello quiere decir que, a mayores niveles de rentabilidad financiera, los niveles de liquidez corriente serán menores en las empresas analizadas que publican información finan- 
ciera en la BVL en el período 2012, esto también se aplica viceversa.

Tabla 8

Análisis de correlación entre rentabilidad financiera y liquidez corriente en el período 2013

\begin{tabular}{lccc}
\hline & \multicolumn{3}{c}{ Liquidez Corriente } \\
\cline { 2 - 4 } & $\mathrm{r}$ & $\mathrm{P}$ & $\mathrm{N}$ \\
\hline $\begin{array}{l}\text { Rentabilidad } \\
\text { Financiera }\end{array}$ & -.186 & .333 & 29 \\
\hline
\end{tabular}

Se observa, en la tabla 8 , el coeficiente de correlación según Pearson es $r=-.186$, que indica la existencia de una correlación negativa inversa y $\mathrm{p}=.333$ lo que señala una correlación no significativa. Ello quiere decir que, a mayores niveles de rentabilidad financiera, los niveles de liquidez corriente serán menores en las empresas analizadas que publican información financiera en la BVL en el período 2013, esto también se aplica viceversa.

\section{Tabla 9}

Análisis de correlación entre rentabilidad financiera y liquidez corriente en el período 2014

\begin{tabular}{lrcc}
\hline & \multicolumn{3}{c}{ Liquidez Corriente } \\
\cline { 2 - 4 } & $\mathrm{r}$ & $\mathrm{P}$ & $\mathrm{N}$ \\
\hline $\begin{array}{l}\text { Rentabilidad } \\
\text { Financiera }\end{array}$ & -.203 & .291 & 29 \\
\hline
\end{tabular}

Se observa, en la tabla 9, el coeficiente de correlación según Pearson es $r=-.203$ que indica la existencia de una correlación negativa inversa y $\mathrm{p}=.291$ lo que señala una correlación no significativa. Ello quiere decir que, a mayores niveles de rentabilidad financiera, los niveles de liquidez corriente también serán mayores en las empresas analizadas que publican información financiera en la BVL en el período 2014, esto también se aplica viceversa.

\section{Conclusiones}

Existe relación $(r=-0.218 ; p=0.019)$ entre la rentabilidad financiera y la liquidez corriente, en las empresas del sector industrial que negocian en la Bolsa de Valores de Lima de los períodos 2011 - 2014. De acuerdo al primer objetivo específico, la cantidad de empresas, según el nivel de rentabilidad financiera, es bajo $30 \%$, medio $41 \%$ y alto $29 \%$, de las empresas que cotizan en la Bolsa de Valores de Lima, período 2011 - 2014. De acuerdo al segundo objetivo específico, la cantidad de empresas, según el nivel de liquidez corriente, es bajo 31\%, medio 39\% y alto 30\%, de las empresas que cotizan en la Bolsa de Valores de Lima, período 2011 - 2014.

\section{Referencias}

Aguilar Pinedo, V. (2013). Gestión de cuentas por cobrar y su incidencia en la liquidez de la empresa contratista. Corporación Petrolera S.A.C. - año 2012, 1-94.

Altuve, G., y José, G. (2014). Rentabilidad de la variable activo corriente, 5-17.

Baena Toro, D. (2014). Finanzas. Igarss 2014. Recuperado de http://doi.org/10.1007/s13398-014-0173-7.2

Baena Tovar, N. (2008). La liquidez en los mercados financieros: repercusiones de la crisis crediticia, 1-53.

Baños Moreno, E.; Pérez Rocha, I. y Vásquez Argueta, A. (2011). Estrategias financieras para minimizar el riesgo de liquidez en las empresas del municipio de San Salvador, dedicadas a la importación y comercialización de piso cerámico. Universidad de El Salvador.

Bustamante, C. (2011). Interpretación de ratios de liquidez. Recuperado de http://www.caballerobustamante.com. pe/plantilla/lab/liquidez_y_rentabilidad.pdf

Castillo Yésica. (2012). Evaluación financiera de la liquidez en las empresas distribuidoras de productos farmacéuticos a través del ciclo de conversión de efectivo.

Flores Díaz, W. (2010). Características del riesgo y eficacia de la rentabilidad en las empresas del sector industrial peruano. Universidad de San Martín de Porras.

Gajardo Bahamonde, T., y Saldivia Díaz, V. (2013). Análisis financiero del subsector de alimentos, bebidas y tabaco de Chile, a través de indicadores financieros 2012. Igarss 2014. Universidad Austral de Chile.

González, M.; Bouzada, A. y Caiafa, M. (2014). Cómo mejorar la rentabilidad de las empresas.

Garcia Restrepo, L. (2014). Liquidez y rentabilidad como factor determinante en el éxito de las empresas, 1-15.

Gitman, l. (2011). Finance 11th ed Solution Lawrence J Gitan Principles of managerial finance 11th, 1-5.

Hunt j. \& media d. (2015). Qué determina la rentabilidad de una empresa, 2015.

José, M., María, M. Q., \& Luís, M. Q. (2008). Relación intertemporal entre rentabilidad y liquidez en el mercado de valores español, 17, 187-204. 
Rentabilidad financiera y liquidez corriente de las empresas del sector industrial que negocian en la Bolsa de Valores de Lima, 2011-2014

Lee, J. K.-Y., \& Wong, A. Y.-T. (2009). Impact of financial liberalization on stock market liquidity: experience of china. Journal of chinese economic and foreign trade studies.

Ley General de Sociedades № 26887 (1997). Recuperado de http://www.lexadin.nl/wlg/legis/nofr/oeur/arch/per/ ley_general_sociedades.pdf

Luengo, P., Antón, M, Sánchez, j., \& Vela, J. (2005). Evaluación de los factores determinantes de la rentabilidad y solvencia de la empresa murciana.

Pascual, M. (2014). Conceptos financieros, 1-5. Recuperado de http://webs.ono.com/martinpascual/pv70601_ tresconceptos.pdf
Rodríguez Castro, D. (2013). Administración del capital de trabajo y su influencia en la rentabilidad en la Empresa Consorcio Roga S.A.C. Trujillo 2011-2012.

Rodríguez Dina. (2013). Administración del capital de trabajo y su influencia en la rentabilidad de la empresa Consorcio Roga S.A.C. Trujillo 2011-2012.

Rubio Domínguez, P. (2007). Análisis de estados financieros. Manual análisis financiero, 1-64. Recuperado de www.eumed.net/libros/2007a/255/

Saucedo L. \& Oyola E. (2014). La administración del capital de trabajo y su influencia en la rentabilidad del club deportivo ABC SA. 